\title{
CONCENTRATIONS MAXIMALES \\ ADMISSIBLES DANS L'AIR, POUR 40 HEURES D'EXPOSITION PAR SEMAINE, DES RADIONUCLÉIDES PRODUITS PAR LA FISSION THERMIQUE DE L'URANIUM 235
}

\section{N. FOURCADE-CANCELLE *}

(manuscrit reçu le is janvier 1969)

\begin{abstract}
RÉSUMÉ
On s'est proposé dans cette étude de déterminer la CMA «air 40 heures » applicable dans la pratique de la radioprotection au mélange des produits de fission formés par irradiation de l'uranium dans un flux thermique. Les durées d'irradiation considérées sont de 30 , roo et 500 jours, et les temps de refroidissement vont de $\mathrm{I}$ jour à 30 ans.

Dans le cas d'une irradiation de 500 jours, par exemple, la CMA va de $5 \cdot 10^{-8} \mathrm{Ci} / \mathrm{m}^{3}$ pour I jour de refroidissement à $3 \cdot 10^{-9}$ pour 30 ans.
\end{abstract}

\section{INTRODUCTION}

Summers et GASKE[r] ont calculé les CMA applicables au mélange des produits provenant de la fission simultanée, par neutrons lents, de 10000 atomes d'uranium 235; il s'agit là d'une irradiation très brève. Pour une irradiation longue allant jusqu'à 3 ans et pour des refroidissements de 2 jours à 4 ans $\mathrm{A}$. BrodSKY [2] a déterminé les doses reçues par différents organes à la suite d'une inhalation correspondant à une exposition de $\mathrm{I}$ Ci.s. ${ }^{-3}$. Une étude analogue faite par G. VACCA [3] donne les doses délivrées à quelques organes après l'absorption de I $\mu \mathrm{Ci}$ de produits de fission provenant d'uranium irradié pendant 300 jours et refroidi de I jour à 3 ans. On s'est proposé ici, de calculer la CMA « air $40 \mathrm{~h}$ " à utiliser dans la pratique de la radioprotection pour le mélange des produits de fission formés par l'irradiation prolongée - pendant 30,100 et 500 jours - d'un combustible en uranium et pour des refroidissements allant de $\mathrm{I}$ jour à 30 ans. Les mélanges considérés sont supposés ne pas contenir d'autres radionucléides que les produits de fission proprement dits.

\section{CALCUL}

Des tableaux tels que les tableaux 2 à 8 , donnant, en fonction des durées d'irradiation (I) et de refroidissement $(\mathrm{R})$, la liste des principaux produits de fission à considérer et l'activité de chacun d'eux dans le mélange, ont été dressés à l'aide des références [4] et [s]. On a calculé ensuite, pour chaque couple (I, R) la CMA du mélange pour chacun des principaux organes

(*) Service Technique d'Etudes de Protection, Centre d'Etudes Nucléaires de Fontenayaux-Roses, B.P. $n^{\circ}$ 6, 92 - Fontenay-aux-Roses. 
(organisme entier, tractus gastro-intestinal, rein, os et poumon); d'après la publication 2 de la C.I.P.R. (IV,8) cette CMA pour l'organe $x$ est :

$$
(\mathrm{CMA})^{x}=\frac{\sum \mathrm{A}_{i}}{\Sigma \frac{\mathrm{A}_{i}}{(\mathrm{CMA})^{x}}}
$$

ou (CMA) ${ }_{i}$ est la CMA du radionucléide $i$ pour l'organe $x$ (donnée par les publications 2 et 6 de la C.I.P.R.), $A_{i}$ l'activité de ce radionucléide dans le mélange, et $\Sigma \mathrm{A}_{i}$ l'activité totale du mélange.

Pour un mélange donné, il est admis que la CMA à retenir est la plus faible des CMA relatives aux différents organes et l'organe correspondant est considéré comme critique pour le mélange.

\section{RÉSULTATS}

Ils sont donnés par le tableau I et la figure jointe. La CMA va de $7 \cdot 10^{-8}$ à $3 \cdot 10^{-9} \mathrm{Ci} / \mathrm{m}^{3}$; elle s'abaisse lorsqu'augmentent les durées d'irradiation et de refroidissement, mais restent très voisines de $3 \cdot 10^{-9} \mathrm{Ci} / \mathrm{m}^{3}$ pour des refroidissements supérieurs à 30 ans. L'organe critique est le poumon pour des refroidissements inférieurs à 200 jours, et l'os pour des refroidissements supérieurs.

\section{COMMENTAIRES}

On n'a retenu, dans les tableaux 4 à 8 , que les produits de fission contribuant chacun pour plus du millième à l'activité du mélange. Les produits négligés, qui sont surtout des radionucléides à vie courte, ont des CMA supérieures en moyenne, à celles des produits retenus. Pour des temps de refroidissement courts, les CMA trouvées sont donc un peu plus sévères que celles qu'on aurait calculées en tenant compte de tous les produits de fission.

Les CMA ainsi déterminées ne s'appliquent qu'au mélange des produits de fission dans les proportions où ils se trouvent dans un combustible irradié. C'est là un cas plutôt idéal, car, le plus souvent, les produits de fission se dégageront dans l'atmosphère dans des proportions très différentes, qui dépendront du processus de libération et de la forme physico-chimique de chaque élément. On peut cependant trouver dans la pratique des conditions assez voisines du cas idéal : par exemple, au cours du traitement de combustibles irradiés, en cas de fuite puis d'évaporation lente de la solution de produits de fission provenant de la première extraction. Par contre, les produits gazeux se sépareront toujours très vite du reste du mélange. Aussi, bien que les kryptons et les xénons figurent dans les tableaux i à 7 , nous n'avons pas tenu compte de ces éléments dans le calcul de la CMA. On peut noter que celle-ci ne s'en trouve qu'environ ro \% plus sévère.

D'après le décret du 20 juin 1966 sur la Protection contre les Rayonnements Ionisants (Annexe IV, Tableau III), la CMA « $40 \mathrm{~h}$ » qui s'appliquerait au mélange des produits de fission ( $5^{\mathrm{e}} \mathrm{cas}$ du tableau III) serait de $3 \cdot 10^{-10} \mathrm{Ci} / \mathrm{m}^{3}$.

Les CMA trouvées ici sont de to à 200 fois moins sévères.

\section{RÉFÉRENCES}

[r] D.L. Summers and M.C. Gaske. Maximum Permissible Activity for Fission Products in Air and Water. Health Pbysics, 1961, vol. 4, no 3/4, pp. 289-292.

[2] A. Brodsky. Criteria for acute exposure to mixed fission product aerosols. Health Pbysics, I965, vol. II, no ro.

[3] G. VAcca. Dose délivrée après une absorption unique d'un mélange de produits de fission. Rapport CEA, n ${ }^{\circ}{ }_{163}$ (1962).

[4] Produits de la fission thermique de l'uranium 235. Rapport SESR/SEPIA, n० 87, 1966. [s] J.O. BLOMEKe and ToDd. ORNL 2127, partie II. 
TABLEAU 1

CMA «AIR 40 H» DU MÉLANGE DES PRINCIPAUX PRODUITS DE FISSION NON GAZEUX DE L'URANIUM 235 PLACÉ DANS UN FLUX DE NEUTRONS THERMIQUES

\begin{tabular}{|c|c|c|c|c|c|}
\hline \multirow{3}{*}{$\begin{array}{c}\text { Temps } \\
\text { d'irradiation } \\
\text { en jours }\end{array}$} & \multirow{3}{*}{$\frac{\text { Temps }}{\text { de }}$} & \multicolumn{3}{|c|}{ Pour I watt d'irradiation } & \multirow{3}{*}{$\begin{array}{c}\text { CMA } \\
\text { " air } 40 \mathrm{~h} \text { » } \\
\text { en } \mathrm{Ci} / \mathrm{m}^{3}\end{array}$} \\
\hline & & \multicolumn{2}{|c|}{$\Sigma A_{i}$ en curies } & \multirow{2}{*}{$\begin{array}{l}\text { Organe } \\
\text { critique }\end{array}$} & \\
\hline & & (1) & $\begin{array}{c}\text { sans } \mathrm{PF} \text { gazeux } \\
\text { (2) }\end{array}$ & & \\
\hline $\begin{array}{r}30 \\
100 \\
500\end{array}$ & I jour & $\begin{array}{l}5,8 \cdot 10^{-1} \\
7,4 \cdot 10^{-1} \\
8,9 \cdot 10^{-1}\end{array}$ & $\begin{array}{l}5,2 \cdot 10^{-1} \\
6,8 \cdot 10^{-1} \\
7,6 \cdot 10^{-1}\end{array}$ & poumon & $\begin{array}{l}7,8 \cdot 10^{-8} \\
7 \cdot 10^{-8} \\
5,2.10^{-8}\end{array}$ \\
\hline $\begin{array}{r}30 \\
100 \\
500\end{array}$ & 10 jours & $\begin{array}{l}2,2.10^{-1} \\
3,7 \cdot 10^{-1} \\
5 \cdot 10^{-1}\end{array}$ & $\begin{array}{l}2.10^{-1} \\
3,4 \cdot 10^{-1} \\
4,8 \cdot 10^{-1}\end{array}$ & poumon & $\begin{array}{l}5,3 \cdot 10^{-8} \\
4,8 \cdot 10^{-8} \\
3,7 \cdot 10^{-8}\end{array}$ \\
\hline $\begin{array}{r}30 \\
100 \\
500\end{array}$ & roo jours & $\begin{array}{l}3,9 \cdot 10^{-2} \\
8,4 \cdot 10^{-2} \\
1,6 \cdot 10^{-1}\end{array}$ & $\begin{array}{l}3,4 \cdot 10^{-2} \\
8,3 \cdot 10^{-2} \\
1,6 \cdot 10^{-1}\end{array}$ & poumon & $\begin{array}{l}3,2 \cdot 10^{-8} \\
2,9 \cdot 10^{-8} \\
2,2 \cdot 10^{-8}\end{array}$ \\
\hline $\begin{array}{r}30 \\
100 \\
500\end{array}$ & $I$ an & $\begin{array}{l}6,8 \cdot 10^{-3} \\
2.10^{-2} \\
4,7 \cdot 10^{-2}\end{array}$ & $\begin{array}{l}4,9 \cdot 10^{-3} \\
1,4 \cdot 10^{-2} \\
4,4 \cdot 10^{-2}\end{array}$ & os & $\begin{array}{l}1,8 \cdot 10^{-8} \\
1,7 \cdot 10^{-8} \\
1,4 \cdot 10^{-8}\end{array}$ \\
\hline $\begin{array}{r}30 \\
100 \\
500\end{array}$ & 3 ans & $\begin{array}{l}1,3 \cdot 10^{-3} \\
4,3 \cdot 10^{-3} \\
1,4 \cdot 10^{-2}\end{array}$ & $\begin{array}{l}1 \cdot 10^{-3} \\
3,2 \cdot 10^{-3} \\
1,3 \cdot 10^{-2}\end{array}$ & os & $\begin{array}{l}7,9 \cdot 10^{-9} \\
7,3 \cdot 10^{-9} \\
6,7 \cdot 10^{-9}\end{array}$ \\
\hline $\begin{array}{r}30 \\
100 \\
500\end{array}$ & Io ans & $\begin{array}{l}4 \cdot 10^{-4} \\
1,3 \cdot 10^{-3} \\
4,5 \cdot 10^{-3}\end{array}$ & $\begin{array}{l}3,5 \cdot 10^{-4} \\
1,2 \cdot 10^{-3} \\
5,4 \cdot 10^{-3}\end{array}$ & os & $\begin{array}{l}4,2 \cdot 10^{-9} \\
4,1 \cdot 10^{-9} \\
3,9 \cdot 10^{-9}\end{array}$ \\
\hline $\begin{array}{r}30 \\
100 \\
500\end{array}$ & 30 ans & $\begin{array}{l}1,7 \cdot 10^{-4} \\
5,7 \cdot 10^{-4} \\
1,8 \cdot 10^{-3}\end{array}$ & $\begin{array}{l}1,5 \cdot 10^{-4} \\
4,9 \cdot 10^{-4} \\
2,4 \cdot 10^{-3}\end{array}$ & os & $\begin{array}{l}3 \cdot 10^{-9} \\
2,9 \cdot 10^{-9} \\
3 \cdot 10^{-9}\end{array}$ \\
\hline
\end{tabular}

(I) Activité totale donnée par J.O. BLOMeke and TodD, ORNL 21 27, partie II.

(2) Résultats des calculs présentés dans les tableaux suivnts. 
R1eure

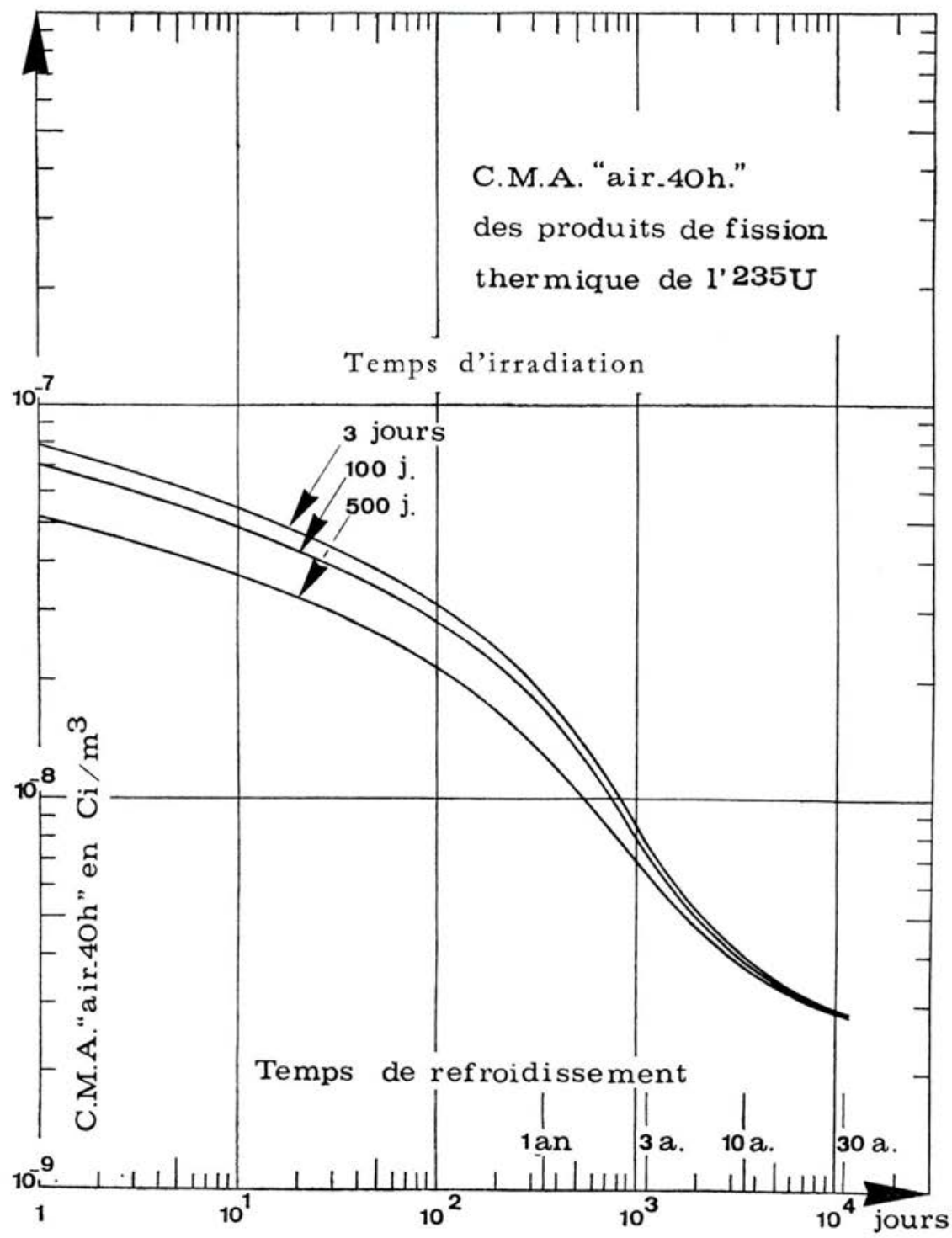


TABLEAU 2

PRINCIPAUX PRODUITS FORMÉS PAR FISSION THERMIQUE DE L'URANIUM 235 ACTIVITÉS BÊTA, EN CURIES,

DANS UN ÉLÉMENT DE COMBUSTIBLE IRRADIÉ A I WATT ET REFROIDI I JOUR

\begin{tabular}{|c|c|c|c|c|c|}
\hline \multirow{2}{*}{ P.F. } & \multirow{2}{*}{ Période } & \multirow{2}{*}{$\begin{array}{c}\text { CMA } \\
\text { " air } 40 \mathrm{~h} \text { " } \\
\text { en } \mathrm{C} i / \mathrm{m}^{3}\end{array}$} & \multicolumn{3}{|c|}{ Temps d'irradiation en jours } \\
\hline & & & 30 & 100 & 500 \\
\hline $\begin{array}{l}85 \mathrm{Kr} \\
85 \mathrm{~m} \mathrm{Kr} \\
88 \mathrm{Kr}\end{array}$ & $\begin{array}{l}\text { I0,4 a } \\
4,4 \mathrm{~h} \\
2,8 \mathrm{~h}\end{array}$ & $\begin{array}{l}\text { 1. Es } \\
\text { 6. E6 }\end{array}$ & $\begin{array}{l}\text { I. Es } \\
2,7 . E_{4} \\
9 . E_{5}\end{array}$ & $\begin{array}{l}\text { 5. Es } \\
2,7 . \mathrm{E}_{4} \\
9 . \mathrm{Es}_{5}\end{array}$ & $\begin{array}{l}\text { 2,2. } \mathrm{E}_{4} \\
\text { 2,7. } \mathrm{E}_{4} \\
\text { 9. } \mathrm{E}_{5}\end{array}$ \\
\hline $88 \mathrm{Rb}$ & $17,8 \mathrm{mn}$ & & I,o. $E_{4}$ & I,o. $\mathrm{E}_{4}$ & $\mathrm{I}, \mathrm{O} . \mathrm{E}_{4}$ \\
\hline $\begin{array}{ll}89 & \mathrm{Sr} \\
90 \mathrm{Sr} \\
91 \mathrm{Sr} \\
92 \mathrm{Sr}\end{array}$ & $\begin{array}{c}51 \mathrm{j} \\
28 \mathrm{a} \\
9,67 \mathrm{~h} \\
2,6 \mathrm{~h}\end{array}$ & $\begin{array}{l}\text { 3. } \mathrm{E}_{8} \\
\text { 1. } \mathrm{E}_{9} \\
\text { 3. } \mathrm{E}_{7} \\
\text { 3. } \mathrm{E}_{7}\end{array}$ & $\begin{array}{l}\text { 1,377. E2 } \\
1,0 . \mathrm{E}_{4} \\
9,62 . \mathrm{E}_{3} \\
9 . \mathrm{E}_{5}\end{array}$ & $\begin{array}{l}\text { 3,036. E2 } \\
3,4 . \mathrm{E}_{4} \\
9,62 . \mathrm{E}_{3} \\
9 . \mathrm{E}\end{array}$ & $\begin{array}{l}\text { 4,044. } E_{2} \\
1,68 \cdot E_{3} \\
9,62 . E_{3} \\
9 . E_{5}\end{array}$ \\
\hline $\begin{array}{ll}90 & \mathrm{Y} \\
9 \mathrm{I} & \mathrm{Y} \\
92 & \mathrm{Y} \\
93 & \mathrm{Y}\end{array}$ & $\begin{array}{c}64,2 \mathrm{~h} \\
58 \mathrm{j} \\
3,6 \mathrm{~h} \\
10 \mathrm{~h}\end{array}$ & $\begin{array}{l}\text { 1. } E_{7} \\
\text { 3. } E_{8} \\
\text { 3. } E_{7} \\
\text { I. } E_{7}\end{array}$ & $\begin{array}{l}9 . \mathrm{E}_{5} \\
1,567 . \mathrm{E}_{2} \\
1,67 . \mathrm{E}_{3} \\
1,119 . \mathrm{E}_{2}\end{array}$ & $\begin{array}{l}3,3 \cdot \mathrm{E}_{4} \\
3,657 \cdot \mathrm{E}_{2} \\
1,67 \cdot \mathrm{E}_{3} \\
1,119 . \mathrm{E}_{2}\end{array}$ & $\begin{array}{l}1,67 . \mathrm{E}_{3} \\
5,280 . \mathrm{E}_{2} \\
1,67 . \mathrm{E}_{3} \\
1,119 . \mathrm{E}_{2}\end{array}$ \\
\hline $\begin{array}{l}95 \mathrm{Nb} \\
96 \mathrm{Nb} \\
97 \mathrm{Nb}\end{array}$ & $\begin{array}{c}35 \mathrm{j} \\
23,4 \mathrm{~h}\end{array}$ & I. $E_{7}$ & $\begin{array}{l}4,33 . \mathrm{E}_{3} \\
6 . \mathrm{E}_{5}\end{array}$ & $\begin{array}{l}2,449 . E_{2} \\
6 . E_{5}\end{array}$ & $\begin{array}{l}5,782 . \mathrm{E}_{2} \\
6 . \mathrm{E}_{5}\end{array}$ \\
\hline $97 \mathrm{Nb}$ & $74 \mathrm{mn}$ & 5. E6 & $2,258 . E_{2}$ & 2,258. E2 & $2,258 . \mathrm{E}_{2}$ \\
\hline $\begin{array}{l}95 \mathrm{Zr} \\
97 \mathrm{Zr}\end{array}$ & $\begin{array}{c}63,3 \mathrm{j} \\
17 \mathrm{~h}\end{array}$ & $\begin{array}{l}\text { 3. E8 } \\
\text { 9. E8 }\end{array}$ & $\begin{array}{l}1,583 . E_{2} \\
2,093 . E_{2}\end{array}$ & $\begin{array}{l}3,791 . E_{2} \\
2,093 . E_{2}\end{array}$ & $\begin{array}{l}5,754 \cdot E_{2} \\
2,093 . E_{2}\end{array}$ \\
\hline 99 Mo & $67 \mathrm{~h}$ & 2. $\mathrm{E}_{7}$ & $4,339 . \mathrm{E}_{2}$ & $4,342 . \mathrm{E}_{2}$ & $4,342 . \mathrm{E}_{2}$ \\
\hline I03 Ru & $4 \mathrm{I} \mathrm{j}$ & 8. E8 & I,029. E2 & 2,089. E2 & 2,537. $\mathrm{E}_{2}$ \\
\hline $\begin{array}{l}\text { I05 Ru } \\
\text { I06 Ru }\end{array}$ & $\begin{array}{c}4,5 \mathrm{~h} \\
\mathrm{I} \text { a }\end{array}$ & $\begin{array}{l}\text { 5. } \mathrm{E}_{7} \\
\text { 6. } \mathrm{E}_{9}\end{array}$ & $\begin{array}{l}\text { I,9. E4 } \\
\text { I,8. E4 }\end{array}$ & $\begin{array}{l}1,9 \cdot E_{4} \\
5,7 \cdot E_{4}\end{array}$ & $\begin{array}{l}1,9 \cdot \mathrm{E}_{4} \\
2,04 \cdot \mathrm{E}_{3}\end{array}$ \\
\hline $\begin{array}{l}\text { ros } \mathrm{Rh} \\
\text { ro6 Rh }\end{array}$ & $\begin{array}{c}36,5 \mathrm{~h} \\
30 \mathrm{~s}\end{array}$ & 5. $\mathrm{E}_{7}$ & $\begin{array}{l}5,78 . E_{3} \\
\mathrm{r}, 8 . E_{4}\end{array}$ & $\begin{array}{l}5,78 \cdot E_{3} \\
5,7 \cdot E_{4}\end{array}$ & $\begin{array}{l}5,78 \cdot E_{3} \\
2,04 \cdot E_{3}\end{array}$ \\
\hline $\begin{array}{l}109 \mathrm{Pd} \\
112 \mathrm{Pd}\end{array}$ & $\begin{array}{l}13,6 \mathrm{~h} \\
21 \mathrm{~h}\end{array}$ & 4. $\mathrm{E}_{7}$ & $\begin{array}{l}\text { 8. Es } \\
\text { s. Es }\end{array}$ & $\begin{array}{l}\text { 8. Es } \\
\text { s. Es }\end{array}$ & $\begin{array}{l}\text { 8. Es } \\
\text { 5. Es }\end{array}$ \\
\hline $\begin{array}{l}\text { III Ag } \\
\text { II } 2 \mathrm{Ag}\end{array}$ & $\begin{array}{l}7,5 \mathrm{j} \\
3,2 \mathrm{~h}\end{array}$ & 2. $\mathrm{E}_{7}$ & $\begin{array}{l}1,3 . \mathrm{E}_{4} \\
6 . \mathrm{Es}_{5}\end{array}$ & $\begin{array}{l}\text { 1,3. E4 } \\
6 . \mathrm{Es}\end{array}$ & $\begin{array}{l}\text { I,3. E4 } \\
6 . \mathrm{E}_{5}\end{array}$ \\
\hline IIs Cd & $53 \mathrm{~h}$ & 2. $E_{7}$ & 6. Es & 6. Es & 6. Es \\
\hline $\begin{array}{l}121 \mathrm{Sn} \\
125 \mathrm{Sn} \\
127 \mathrm{Sn}\end{array}$ & $\begin{array}{l}27,5 \mathrm{~h} \\
9,5 \mathrm{j} \\
1,5 \mathrm{~h}\end{array}$ & 8. E8 & $\begin{array}{l}\text { 6. Es } \\
1,6 . E_{4} \\
1,89 . E_{3}\end{array}$ & $\begin{array}{l}\text { 6. Es } \\
1,8 . E_{4} \\
1,89 . E_{3}\end{array}$ & $\begin{array}{l}\text { 6. Es } \\
1,8 . E_{4} \\
1,89 . E_{3}\end{array}$ \\
\hline
\end{tabular}

Nota - I. Es doit se lire $1.10^{-5}$. 
TABLEAU 2 (suite)

PRINCIPAUX PRODUITS FORMÉS PAR FISSION THERMIQUE DE L'URANIUM 235 ACTIVITÉS BÊTA, EN CURIES, DANS UN ÉLÉMENT DE COMBUSTIBLE IRRADIÉ A I WATT ET REFROIDI I JOUR

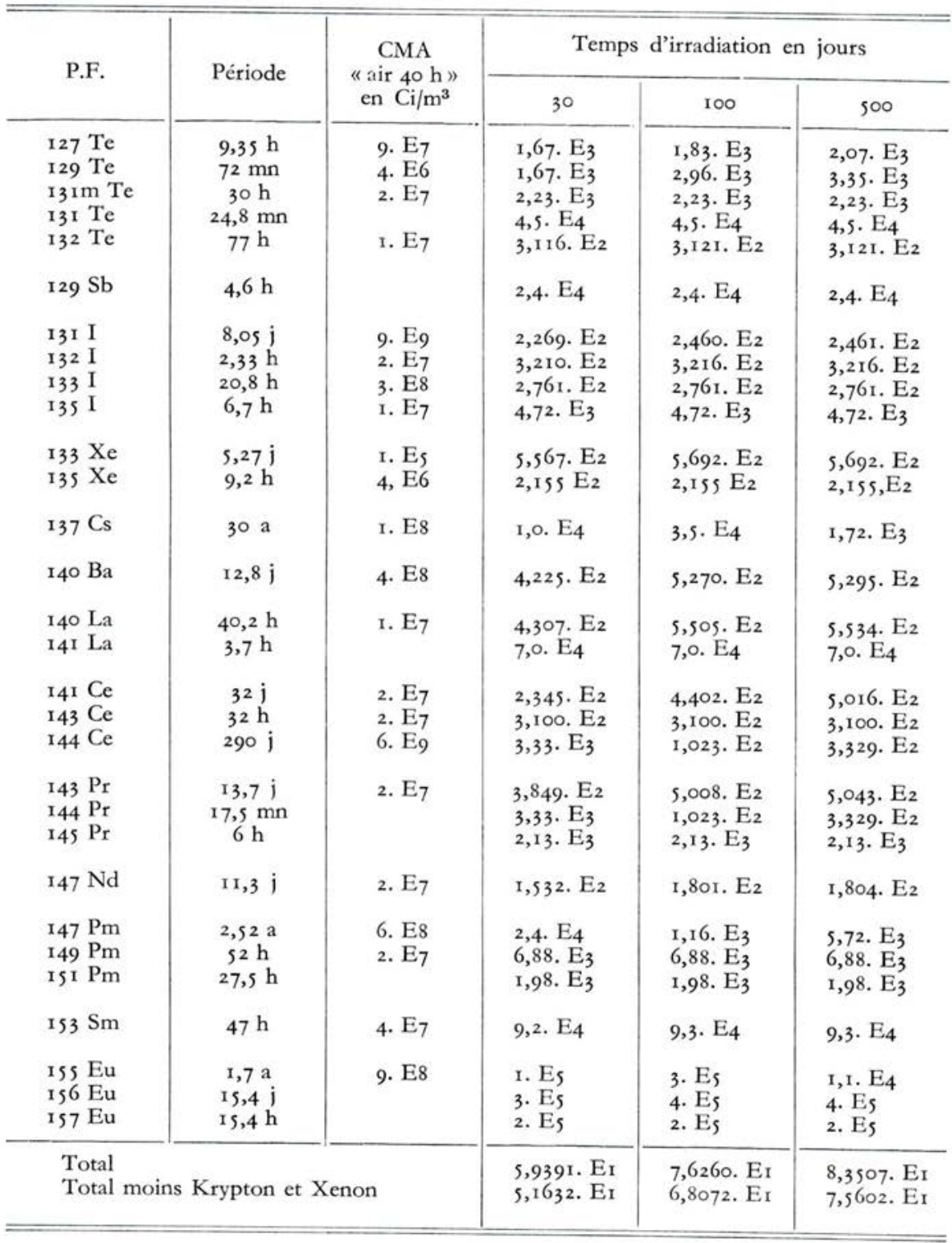

Nota - 4. E8 doit se lire $4 \cdot 10^{-8}$. 
TABLEAU 3

PRINCIPAUX PRODUITS FORMÉS PAR FISSION THERMIQUE DE L'URANIUM 235 ACTIVITÉS BÊTA, EN CURIES,

DANS UN ÉLÉMENT DE COMBUSTIBLE IRRADIÉ A I WATT ET REFROIDI IO JOURS

\begin{tabular}{|c|c|c|c|c|c|}
\hline \multirow{2}{*}{ P.F. } & \multirow{2}{*}{ Période } & \multirow{2}{*}{$\begin{array}{c}\text { CMA } \\
\text { " air } 40 \mathrm{~h} \text { » } \\
\text { en } \mathrm{Ci} / \mathrm{m}^{3}\end{array}$} & \multicolumn{3}{|c|}{ Temps d'irradiation en jours } \\
\hline & & & 30 & 100 & 500 \\
\hline 8 s $\mathrm{Kr}$ & 10,4 a & I. Es & I. Es & s. Es & $2,2 . \mathrm{E}_{4}$ \\
\hline $\begin{array}{l}89 \mathrm{Sr} \\
90 \mathrm{Sr}\end{array}$ & $\begin{array}{l}51 \mathrm{j} \\
28 \mathrm{a}\end{array}$ & $\begin{array}{l}\text { 3. E8 } \\
\text { I. E9 }\end{array}$ & $\begin{array}{l}1,216 . E_{2} \\
1,0 E_{4}\end{array}$ & $\begin{array}{l}2,680 . E_{2} \\
3,4 \cdot E_{4}\end{array}$ & $\begin{array}{l}3,570 . \mathrm{E}_{2} \\
\mathrm{I}, 68 . \mathrm{E}_{3}\end{array}$ \\
\hline $\begin{array}{l}90 \mathrm{Y} \\
91 \mathrm{Y}\end{array}$ & $\begin{array}{c}64,2 \mathrm{~h} \\
58 \mathrm{j}\end{array}$ & $\begin{array}{l}\text { 1. } \mathrm{E}_{7} \\
\text { 3. } \mathrm{E}_{8}\end{array}$ & $\begin{array}{l}\text { 1,o. } E_{4} \\
1,416 . E_{2}\end{array}$ & $\begin{array}{l}3,4 \cdot \mathrm{E}_{4} \\
3,296 . \mathrm{E}_{2}\end{array}$ & $\begin{array}{l}1,66 . \mathrm{E}_{3} \\
4,756 . \mathrm{E}_{2}\end{array}$ \\
\hline $95 \mathrm{Zr}$ & $63,3 \mathrm{j}$ & 3. E8 & $1,438 . E_{2}$ & $3,444 . E_{2}$ & $5,227 \cdot \mathrm{E}_{2}$ \\
\hline $95 \mathrm{Nb}$ & $35 \mathrm{j}$ & I. $\mathrm{E}_{7}$ & $6,08 . E_{3}$ & $2,639 . \mathrm{E}_{2}$ & $5,733 \cdot \mathrm{E}_{2}$ \\
\hline $99 \mathrm{Mo}$ & $67 \mathrm{~h}$ & 2. $\mathrm{E}_{7}$ & ${ }_{4,65} \cdot \mathrm{E}_{3}$ & ${ }_{4}, 65 . \mathrm{E}_{3}$ & ${ }_{4,65} . \mathrm{E}_{3}$ \\
\hline $\begin{array}{l}103 \mathrm{Ru} \\
106 \mathrm{Ru}\end{array}$ & $\begin{array}{l}4 \mathrm{I} j \\
\mathrm{I} \text { a }\end{array}$ & $\begin{array}{l}\text { 8. E8 } \\
\text { 6. E9 }\end{array}$ & $\begin{array}{l}8,80 . \mathrm{E}_{3} \\
\mathrm{I}, 8 . \mathrm{E}_{4}\end{array}$ & $\begin{array}{l}1,787 . \mathrm{E}_{2} \\
5,6 \cdot \mathrm{E}_{4}\end{array}$ & $\begin{array}{l}2,171 \cdot E_{2} \\
2,01 \cdot E_{3}\end{array}$ \\
\hline $106 \mathrm{Rh}$ & $30 \mathrm{~s}$ & & I,8. $\mathrm{E}_{4}$ & $5,6 . \mathrm{E}_{4}$ & $2, \mathrm{OI} \cdot \mathrm{E}_{3}$ \\
\hline III Ag & $7,5 \mathrm{j}$ & 2. $\mathrm{E}_{7}$ & s. Es & s. Es & s.Es \\
\hline $\begin{array}{l}125 \mathrm{Sn} \\
127 \mathrm{Sn}\end{array}$ & $\begin{array}{l}9,5 \mathrm{j} \\
\mathrm{I}, 5 \mathrm{~h}\end{array}$ & 8. E. 8 & $\begin{array}{l}\text { 8. Es } \\
3,4 . \mathrm{E}_{4}\end{array}$ & $\begin{array}{l}\text { 9. Es } \\
3,5 . \mathrm{E}_{4}\end{array}$ & $\begin{array}{l}9 . \mathrm{Es}_{5} \\
3,5 . \mathrm{E}_{4}\end{array}$ \\
\hline $\begin{array}{l}127 \mathrm{Te} \\
129 \mathrm{Te} \\
132 \mathrm{Te}\end{array}$ & $\begin{array}{l}9,35 \mathrm{~h} \\
72 \mathrm{mn} \\
77 \mathrm{~h}\end{array}$ & $\begin{array}{l}\text { 9. } \mathrm{E}_{7} \\
\text { 4. } \mathrm{E}_{6} \\
\text { 1. } \mathrm{E}_{7}\end{array}$ & $\begin{array}{l}3,9 \cdot E_{4} \\
1,22 \cdot E_{3} \\
4,57 \cdot E_{3}\end{array}$ & $\begin{array}{l}5,3 \cdot E_{4} \\
2,28 \cdot E_{3} \\
4,58 \cdot E_{3}\end{array}$ & $\begin{array}{l}7,5 \cdot \mathrm{E}_{4} \\
2,60 \cdot \mathrm{E}_{3} \\
4,58 \cdot \mathrm{E}_{3}\end{array}$ \\
\hline $\begin{array}{l}131 \\
132\end{array}$ & $\begin{array}{l}8,05 \mathrm{j} \\
2,33 \mathrm{~h}\end{array}$ & $\begin{array}{l}\text { 9. } \mathrm{E}_{9} \\
\text { 2. } \mathrm{E}_{7}\end{array}$ & $\begin{array}{l}1,068 \cdot E_{2} \\
4,71 . E_{3}\end{array}$ & $\begin{array}{l}\mathrm{I}, \mathrm{I} 57 . \mathrm{E}_{2} \\
4,72 . \mathrm{E}_{3}\end{array}$ & $\begin{array}{l}1,157 . E_{2} \\
4,72 . E_{3}\end{array}$ \\
\hline $133 \mathrm{Xe}$ & $5,27 \mathrm{j}$ & I. $\mathrm{E}_{3}$ & 1,909. E2 & I,948. E2 & I,948. E2 \\
\hline $137 \mathrm{Cs}$ & $30 \mathrm{a}$ & I. E8 & $\mathrm{I}, 1 . \mathrm{E}_{4}$ & $3,5 \cdot \mathrm{E}_{4}$ & $1,72 . E_{3}$ \\
\hline $14 \circ \mathrm{Ba}$ & $12,8 \mathrm{j}$ & 4. E8 & $2,615 . E_{2}$ & 3,262. $\mathrm{E}_{2}$ & $3,277 . \mathrm{E}_{2}$ \\
\hline I $40 \mathrm{La}$ & $40,2 \mathrm{~h}$ & I. $\mathrm{E}_{7}$ & $2,987 . \mathrm{E}_{2}$ & $3,728 . \mathrm{E}_{2}$ & $3,747 \cdot \mathrm{E}_{2}$ \\
\hline $\begin{array}{l}14 \mathrm{I} \mathrm{Ce} \\
143 \mathrm{Ce} \\
\text { I } 44 \mathrm{Ce}\end{array}$ & $\begin{array}{l}32 \mathrm{j} \\
32 \mathrm{~h} \\
290 \mathrm{j}\end{array}$ & $\begin{array}{l}\text { 2. } \mathrm{E}_{7} \\
\text { 2. } \mathrm{E}_{7} \\
\text { 6. } \mathrm{E}_{9}\end{array}$ & $\begin{array}{l}1,944 \cdot \mathrm{E}_{2} \\
3,3 \cdot \mathrm{E}_{4} \\
3,26 \cdot \mathrm{E}_{3}\end{array}$ & $\begin{array}{l}3,644 \cdot \mathrm{E}_{2} \\
3,3 \cdot \mathrm{E}_{4} \\
1, \mathrm{OOI} \cdot \mathrm{E}_{2}\end{array}$ & $\begin{array}{l}4, \mathrm{I} 53 \cdot \mathrm{E}_{2} \\
3,3 \cdot \mathrm{E}_{4} \\
3,257 \cdot \mathrm{E}_{2}\end{array}$ \\
\hline $\begin{array}{l}\text { I } 43 \mathrm{Pr} \\
\text { I } 44 \mathrm{Pr}\end{array}$ & $\begin{array}{c}13,7 \mathrm{j} \\
17,5 \mathrm{mn}\end{array}$ & 2. $\mathrm{E}_{7}$ & $\begin{array}{l}2,664 \cdot \mathrm{E}_{2} \\
3,26 \cdot \mathrm{E}_{3}\end{array}$ & $\begin{array}{l}3.401 . E_{2} \\
1,001 . E_{2}\end{array}$ & $\begin{array}{l}3,424 \cdot \mathrm{E}_{2} \\
3,257 \cdot \mathrm{E}_{2}\end{array}$ \\
\hline I $47 \mathrm{Nd}$ & $11,3 j$ & 2. $\mathrm{E}_{7}$ & $8,69 . \mathrm{E}_{3}$ & $1,022 . E_{2}$ & $\mathrm{I}, 023 . \mathrm{E}_{2}$ \\
\hline $\begin{array}{l}\text { I } 47 \mathrm{Pm} \\
\text { I } 49 \mathrm{Pm}\end{array}$ & $\begin{array}{l}2,52 \mathrm{a} \\
52 \mathrm{~h}\end{array}$ & $\begin{array}{l}\text { 6. } \mathrm{E}_{8} \\
\text { 2. } \mathrm{E}_{7}\end{array}$ & $\begin{array}{l}3,2 . E_{4} \\
4,1 . E_{4}\end{array}$ & $\begin{array}{l}1,24 \cdot E_{3} \\
4,1 \cdot E_{4}\end{array}$ & $\begin{array}{l}5,77 \cdot E_{3} \\
4,1 \cdot E_{4}\end{array}$ \\
\hline $\begin{array}{l}\text { I } 55 \mathrm{Eu} \\
\text { is } 6 \mathrm{Eu}\end{array}$ & $\begin{array}{l}1,7 \text { a } \\
15,4 j\end{array}$ & 7. E8 & $\begin{array}{l}\text { 1. Es } \\
\text { 2. Es }\end{array}$ & $\begin{array}{l}\text { 3. Es } \\
\text { 3. Es }\end{array}$ & $\begin{array}{l}\text { 1, 1. E4 } \\
\text { 3. Es }\end{array}$ \\
\hline \multicolumn{3}{|c|}{$\begin{array}{l}\text { Total } \\
\text { Total } \mathrm{m}\end{array}$} & $\begin{array}{l}2,2044 \cdot E_{I} \\
2,0 I_{34} \text {. EI }\end{array}$ & $\begin{array}{l}3,6159 . E_{1} \\
3,4206 . E_{I}\end{array}$ & $\begin{array}{l}5,0069 . E_{1} \\
4,8099 . E_{1}\end{array}$ \\
\hline
\end{tabular}

Nota - I. Es doit se lire $1 \cdot 10^{-5}$. Nota - 4. E8 doit se lire 4.10-8. 


\section{TABLEAU 4}

PRINCIPAUX PRODUITS FORMÉS PAR FISSION THERMIQUE DE L'URANIUM 235 ACTIVITÉ BÊTA, EN CURIES,

DANS UN ÉLÉMENT DE COMBUSTIBLE IRRADIÉ A I WATT ET REFROIDI IOO JOURS

\begin{tabular}{|c|c|c|c|c|c|}
\hline \multirow{2}{*}{ P.F. } & \multirow{2}{*}{ Période } & \multirow{2}{*}{$\begin{array}{c}\text { CMA } \\
\text { « air } 40 \mathrm{~h} \text { » } \\
\text { en } \mathrm{C}: / \mathrm{m}^{3}\end{array}$} & \multicolumn{3}{|c|}{ Temps d'irradiation en jours } \\
\hline & & & 30 & 100 & 500 \\
\hline $85 \mathrm{Kr}$ & I0,4 a & I. Es & I. Es & s. Es & 2,2. $E_{4}$ \\
\hline $\begin{array}{l}89 \mathrm{Sr} \\
90 \mathrm{Sr}\end{array}$ & $\begin{array}{ll}5 \mathrm{I} & \mathrm{j} \\
28 & \mathrm{a}\end{array}$ & $\begin{array}{l}\text { 3. E8 } \\
\text { 1. E9 }\end{array}$ & $\begin{array}{l}3,49 . E_{3} \\
1,0 . E_{4}\end{array}$ & $\begin{array}{l}7,70 \cdot \mathrm{E}_{3} \\
3,4 \cdot \mathrm{E}_{4}\end{array}$ & $\begin{array}{l}1,025 \cdot E_{2} \\
1,67 \cdot E_{3}\end{array}$ \\
\hline $\begin{array}{l}90 \mathrm{Y} \\
9 \mathrm{I} Y\end{array}$ & $\begin{array}{c}64,2 \mathrm{~h} \\
58 \mathrm{j}\end{array}$ & $\begin{array}{l}\text { 1. } \mathrm{E}_{7} \\
\text { 3. } \mathrm{E}_{8}\end{array}$ & $\begin{array}{l}\text { 1,o. } \mathrm{E}_{4} \\
4,92 . \mathrm{E}_{3}\end{array}$ & $\begin{array}{l}3,4 \cdot \mathrm{E}_{4} \\
\mathrm{I}, \mathrm{I} 45 \cdot \mathrm{E}_{2}\end{array}$ & $\begin{array}{l}1,67 \cdot E_{3} \\
1,653 . E_{2}\end{array}$ \\
\hline $95 \mathrm{Zr}$ & $63,3 j$ & 3. E8 & $5,5 \mathrm{I} \cdot \mathrm{E}_{3}$ & ${ }_{1,319 .} \mathrm{E}_{2}$ & 2,002. $\mathrm{E}_{2}$ \\
\hline $95 \mathrm{Nb}$ & $35 \mathrm{i}$ & I. $\mathrm{E}_{7}$ & $7,72 . \mathrm{E}_{3}$ & 2,049. E2 & $3,400 . \mathrm{E}_{2}$ \\
\hline $\begin{array}{l}\text { I03 Ru } \\
\text { I06 Ru }\end{array}$ & $\begin{array}{c}4 \mathrm{I} j \\
\mathrm{I} \text { a }\end{array}$ & $\begin{array}{l}\text { 8. E8 } \\
\text { 6. E9 }\end{array}$ & $\begin{array}{l}1,85 \cdot E_{3} \\
1,5 \cdot E_{4}\end{array}$ & $\begin{array}{l}3,76 \cdot \mathrm{E}_{3} \\
4,8 \cdot \mathrm{E}_{4}\end{array}$ & $\begin{array}{l}4,57 \cdot E_{3} \\
1,70 \cdot E_{3}\end{array}$ \\
\hline $106 \mathrm{Rh}$ & $30 \mathrm{~s}$ & & $\mathrm{I}, 5 \cdot \mathrm{E}_{4}$ & ${ }_{4}, 8 . \mathrm{E}_{4}$ & $\mathrm{I}, 7 \cdot \mathrm{E}_{3}$ \\
\hline $\begin{array}{l}\text { I } 27 \mathrm{Te} \\
\text { I } 29 \mathrm{Te}\end{array}$ & $\begin{array}{l}9,35 \mathrm{~h} \\
72 \mathrm{mn}\end{array}$ & $\begin{array}{l}\text { 9. } \mathrm{E}_{7} \\
\text { 4. } \mathrm{E}_{6}\end{array}$ & $\begin{array}{l}\text { s. Es } \\
1,8 . E_{4}\end{array}$ & $\begin{array}{l}\mathrm{r}, 2 \cdot \mathrm{E}_{4} \\
3,5 \cdot \mathrm{E}_{4}\end{array}$ & $\begin{array}{l}2,5 \cdot E_{4} \\
3,9 \cdot E_{4}\end{array}$ \\
\hline $137 \mathrm{Cs}$ & $30 \mathrm{a}$ & I. E8 & I,o. $E_{4}$ & $3,5 \cdot \mathrm{E}_{4}$ & $\mathrm{I}, 7 \mathrm{I} \cdot \mathrm{E}_{3}$ \\
\hline $\mathrm{I} 40 \mathrm{Ba}$ & $12,8 j$ & 4. E8 & 2,2. $\mathrm{E}_{4}$ & $2,7 \cdot \mathrm{E}_{4}$ & $2,7 \cdot \mathrm{E}_{4}$ \\
\hline $140 \mathrm{La}$ & $40,2 \mathrm{~h}$ & I. $\mathrm{E}_{7}$ & $2,5 \cdot E_{4}$ & $3, \mathrm{I} \cdot \mathrm{E}_{4}$ & $3, \mathrm{I} \cdot \mathrm{E}_{4}$ \\
\hline $\begin{array}{l}\text { I } 4 \mathrm{I} C \mathrm{Ce} \\
\text { I } 44 \mathrm{Ce}\end{array}$ & $\begin{array}{c}32 \mathrm{j} \\
290 \mathrm{j}\end{array}$ & $\begin{array}{l}\text { 2. } \mathrm{E}_{7} \\
\text { 6. } \mathrm{E}_{9}\end{array}$ & $\begin{array}{l}2,93 \cdot E_{3} \\
2,62 . E_{3}\end{array}$ & $\begin{array}{l}5,51 \cdot E_{3} \\
8,03 \cdot E_{3}\end{array}$ & $\begin{array}{l}6,27 . \mathrm{E}_{3} \\
2,615 . \mathrm{E}_{2}\end{array}$ \\
\hline $\begin{array}{l}\text { I } 43 \mathrm{Pr} \\
\text { I } 44 \mathrm{Pr}\end{array}$ & $\begin{array}{c}13,7 \mathrm{j} \\
17,5 \mathrm{mn}\end{array}$ & 2. $\mathrm{E}_{7}$ & $\begin{array}{l}2,9 \cdot E_{4} \\
2,62 . E_{3}\end{array}$ & $\begin{array}{l}3,7 \cdot \mathrm{E}_{4} \\
8,03 \cdot \mathrm{E}_{3}\end{array}$ & $\begin{array}{l}3,7 \cdot \mathrm{E}_{4} \\
2,615 . \mathrm{E}_{2}\end{array}$ \\
\hline I $47 \mathrm{Nd}$ & $\mathrm{I} I, 3 \mathrm{j}$ & 2. $\mathrm{E}_{7}$ & 3.Es & 4. Es & 4. Es \\
\hline${ }_{147} \mathrm{Pm}$ & 2,52 a & 6. E8 & $3,9 . \mathrm{E}_{4}$ & ${ }_{1,27} \cdot E_{3}$ & $5,52 . E_{3}$ \\
\hline Iss Eu & $\mathrm{I}, 7 \mathrm{a}$ & 7. $\mathrm{E} 8$ & & 2. Es & I,o. $E_{4}$ \\
\hline \multicolumn{3}{|c|}{$\begin{array}{l}\text { Total } \\
\text { Total moins Krypton }\end{array}$} & $\begin{array}{l}3,368 \cdot \mathrm{E}_{2} \\
3,367 \cdot \mathrm{E}_{2}\end{array}$ & $\begin{array}{l}8,343 \cdot \mathrm{E}_{2} \\
8,338 \cdot \mathrm{E}_{2}\end{array}$ & $\begin{array}{l}\text { I,6003. EI } \\
1,598 \mathrm{r} \cdot \mathrm{EI}_{\mathrm{I}}\end{array}$ \\
\hline
\end{tabular}

Nota - 2. E7 doit se lire $2 \cdot 10^{-7}$. 


\section{TABLEAU 5}

PRINCIPAUX PRODUITS FORMÉS PAR FISSION THERMIQUE DE L'URANIUM 235 ACTIVITÉS BÊTA, EN CURIES,

DANS UN ÉLÉMENT DE COMBUSTIBLE IRRADIÉ A I WATT ET REFROIDI I AN

\begin{tabular}{|c|c|c|c|c|c|}
\hline \multirow{2}{*}{ P.F. } & \multirow{2}{*}{ Période } & \multirow{2}{*}{$\begin{array}{c}\text { CMA } \\
\text { " air } 40 \mathrm{~h} \text { » } \\
\text { en } \mathrm{C} i / \mathrm{m}^{3}\end{array}$} & \multicolumn{3}{|c|}{ Temps d'irradiation en jours } \\
\hline & & & 30 & 100 & 500 \\
\hline $8 s \mathrm{Kr}$ & Io,4 a & 1. Es & 1.3. Es & 4. Es & $2, \mathrm{I} \cdot \mathrm{E}_{4}$ \\
\hline $\begin{array}{l}89 \mathrm{Sr} \\
90 \mathrm{Sr}\end{array}$ & $\begin{array}{l}5 \mathrm{I} \mathrm{j} \\
28 \mathrm{a}\end{array}$ & $\begin{array}{l}\text { 3. E8 } \\
\text { I. E9 }\end{array}$ & $\begin{array}{l}8,8 \cdot \mathrm{Es}_{5} \\
1, \infty 0 . \mathrm{E}_{4}\end{array}$ & $\begin{array}{l}1,9 \cdot \mathrm{E}_{4} \\
3,3 \cdot \mathrm{E}_{4}\end{array}$ & $\begin{array}{l}2,6 . \mathrm{E}_{4} \\
1,64 \cdot \mathrm{E}_{3}\end{array}$ \\
\hline $\begin{array}{l}90 \mathrm{Y} \\
91 \mathrm{Y}\end{array}$ & $\begin{array}{c}64,2 \mathrm{~h} \\
5^{8} \mathrm{j}\end{array}$ & $\begin{array}{l}\text { I. } \mathrm{E}_{7} \\
\text { 3. } \mathrm{E} 8\end{array}$ & $\begin{array}{l}1,00 \mathrm{E}_{4} \\
2,17 \cdot \mathrm{E}_{4}\end{array}$ & $\begin{array}{l}3,3 \cdot \mathrm{E}_{4} \\
\text { 5,I. } \mathrm{E}_{4}\end{array}$ & $\begin{array}{l}1,64 \cdot \mathrm{E}_{3} \\
7, \cdot 3 \mathrm{E}_{4}\end{array}$ \\
\hline $95 \mathrm{Zr}$ & $63,3 j$ & 3. E8 & $3,25 . E_{4}$ & $7,8 . \mathrm{E}_{4}$ & $\mathrm{I}, \mathrm{I} 8 . \mathrm{E}_{3}$ \\
\hline 95 $\mathrm{Nb}$ & $35 \mathrm{j}$ & I. $E_{7}$ & $6,83 \cdot E_{4}$ & $1,65 . \mathrm{E}_{3}$ & $2,52 . \mathrm{E}_{3}$ \\
\hline $\begin{array}{l}103 \mathrm{Ru} \\
\text { I06 Ru }\end{array}$ & $\begin{array}{l}4 \mathrm{I} j \\
\mathrm{I} \text { a }\end{array}$ & $\begin{array}{l}\text { 8. E8 } \\
\text { 6. E9 }\end{array}$ & $\begin{array}{l}1,9 \cdot \text { Es } \\
9,3 \cdot \text { Es }\end{array}$ & $\begin{array}{l}\text { 4. Es } \\
2,9 . \mathrm{E}_{4}\end{array}$ & $\begin{array}{l}5 . \mathrm{Es}_{5} \\
\mathrm{I}, \mathrm{O} 4 \cdot \mathrm{E}_{3}\end{array}$ \\
\hline Io6 Rh & $30 \mathrm{~s}$ & & $9,3 . \mathrm{Es}_{5}$ & $2,9 . \mathrm{E}_{4}$ & $1,04 \cdot E_{3}$ \\
\hline $127 \mathrm{Te}$ & $9,35 \mathrm{~h}$ & 9. $\mathrm{E}_{7}$ & 8. E6 & 2. Es & 4. Es \\
\hline $137 \mathrm{Cs}$ & $30 \mathrm{a}$ & I. E8 & $\mathrm{I}, \mathrm{O} 2 . \mathrm{E}_{4}$ & $3,4 \cdot E_{4}$ & $1,68 . \mathrm{E}_{3}$ \\
\hline $\begin{array}{l}\text { I } 4 \mathrm{I} \mathrm{Ce} \\
\text { 1 } 44 \mathrm{Ce}\end{array}$ & $\begin{array}{l}32 \mathrm{j} \\
290 \mathrm{j}\end{array}$ & $\begin{array}{l}\text { 2. } \mathrm{E}_{7} \\
\text { 6. } \mathrm{E}_{9}\end{array}$ & $\begin{array}{l}\text { I, I. Es } \\
1,370 . E_{3}\end{array}$ & $\begin{array}{l}\text { 2. Es } \\
4,20 . E_{3}\end{array}$ & $\begin{array}{l}\text { 2. Es } \\
1,368 . E_{2}\end{array}$ \\
\hline${ }_{144} \operatorname{Pr}$ & $17,5 \mathrm{mn}$ & & I,370. $\mathrm{E}_{3}$ & $4,20 . \mathrm{E}_{3}$ & $\mathrm{I}, 368 . \mathrm{E}_{2}$ \\
\hline${ }_{147} \mathrm{Pm}$ & 2,52 a & 6. E8 & $3,22 . \mathrm{E}_{4}$ & $1,05 . E_{3}$ & $4,55 \cdot E_{3}$ \\
\hline Iss Eu & $\mathrm{x}, 7 \mathrm{a}$ & 7. E8 & 3. E6 & 1. Es & 7. Es \\
\hline \multicolumn{3}{|c|}{$\begin{array}{l}\text { Total } \\
\text { Total moins Krypton }\end{array}$} & $\begin{array}{l}4,917 \cdot E_{3} \\
4,904 \cdot E_{3}\end{array}$ & $\begin{array}{l}\mathrm{I}, 429 \cdot \mathrm{E}_{2} \\
\mathrm{I}, 425 \cdot \mathrm{E}_{2}\end{array}$ & $\begin{array}{l}4,403 \cdot \mathrm{E}_{2} \\
4,382 \cdot \mathrm{E}_{2}\end{array}$ \\
\hline
\end{tabular}

Nota - I. Es doit se lire $1.10^{-5}$. 
TABLEAU 6

PRINCIPAUX PRODUITS FORMÉS PAR FISSION THERMIQUE DE L'URANIUM 235 ACTIVITÉS BÊTA, EN CURIES

DANS UN ÉLÉMENT DE COMBUSTIBLE IRRADIÉ A I WATT ET REFROIdI 3 ANS

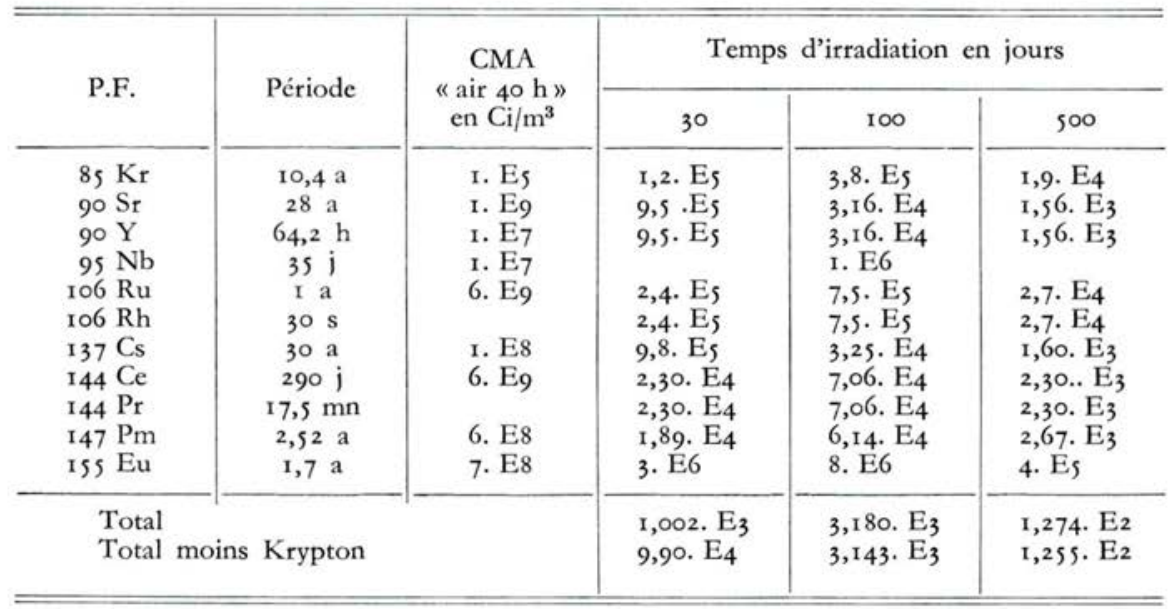

Nota - r. Es doit se lire $1.10^{-5}$.

\section{TABLEAU 7}

PRINCIPAUX PRODUITS DE FISSION THERMIQUE DE L'URANIUM 235 ACTIVITÉS BÊTA, EN CURIES,

DANS UN ÉLÉMENT DE COMBUSTIBLE IRRADIÉ A I WATT ET REFROIDI IO ANS

\begin{tabular}{|c|c|c|c|c|c|}
\hline \multirow{2}{*}{ P.F. } & \multirow{2}{*}{ Période } & \multirow{2}{*}{$\begin{array}{c}\text { CMA } \\
\text { « air } 40 \mathrm{~h} » \\
\text { en } \mathrm{Ci} / \mathrm{m}^{3}\end{array}$} & \multicolumn{3}{|c|}{ Temps d'irradiation en jours } \\
\hline & & & 30 & 100 & 500 \\
\hline $\begin{array}{l}85 \mathrm{Kr} \\
90 \mathrm{Sr} \\
90 \mathrm{Y} \\
\text { r } 37 \mathrm{Cs} \\
\text { r } 44 \mathrm{Ce} \\
144 \mathrm{Pr} \\
\text { I } 47 \mathrm{Pm}\end{array}$ & $\begin{array}{c}10,4 \mathrm{a} \\
28 \mathrm{a} \\
64,2 \mathrm{~h} \\
30 \mathrm{a} \\
290 \mathrm{j} \\
17,5 \mathrm{mn} \\
2,52 \mathrm{a}\end{array}$ & $\begin{array}{l}\text { I. Es } \\
\text { 1. E9 } \\
\text { 1. E8 } \\
\text { 6. E9 }\end{array}$ & $\begin{array}{l}\text { 7. E6 } \\
8,0 . \text { Es } \\
\text { 8,o. Es } \\
\text { 8,3. Es } \\
\text { s,o. Es } \\
\text { s,o. Es } \\
\text { 1,5. Es }\end{array}$ & $\begin{array}{l}2,3 \cdot \mathrm{Es}_{5} \\
2,66 \cdot \mathrm{E}_{4} \\
2,66 \cdot \mathrm{E}_{4} \\
2,75 \cdot \mathrm{E}_{4} \\
1,54 \cdot \mathrm{E}_{4} \\
1,54 \cdot \mathrm{E}_{4} \\
7,6 . \mathrm{E}_{5}\end{array}$ & $\begin{array}{l}\text { 1, 1 2. E } E_{4} \\
1,308 . E_{3} \\
1,308 . E_{3} \\
1,360 . E_{3} \\
5,00 . E_{4} \\
5,00 . E_{4} \\
3,77 . E_{4}\end{array}$ \\
\hline \multicolumn{3}{|c|}{$\begin{array}{l}\text { Total } \\
\text { Total moins Krypton }\end{array}$} & $\begin{array}{l}3,65 \cdot \mathrm{E}_{4} \\
3,52 \cdot \mathrm{E}_{4}\end{array}$ & $\begin{array}{l}1,214 \cdot E_{3} \\
1,191 \cdot E_{3}\end{array}$ & $\begin{array}{l}5,465 \cdot E_{3} \\
5,353 \cdot E_{3}\end{array}$ \\
\hline
\end{tabular}

Nota - I. Es doit se lire $1.10^{-5}$. 
TABLEAU 8

PRINCIPAUX PRODUITS FORMÉS PAR FISSION THERMIQUE DE L'URANIUM 235 ACTIVITÉS BÊTA, EN CURIES,

DANS UN ÉLÉMENT DE COMBUSTIBLE IRRADIÉ A I WATT ET REFROIDI 30 ANS

\begin{tabular}{|c|c|c|c|c|c|}
\hline \multirow{2}{*}{ P.F. } & \multirow{2}{*}{ Période } & \multirow{2}{*}{$\begin{array}{c}\text { CMA } \\
\text { " air } 40 \mathrm{~h} \text { " } \\
\text { en } \mathrm{Ci} / \mathrm{m}^{3}\end{array}$} & \multicolumn{3}{|c|}{ Temps d'irradiation en jours } \\
\hline & & & 30 & 100 & 500 \\
\hline $\begin{array}{c}90 \mathrm{Sr} \\
90 \mathrm{Y} \\
137 \mathrm{Cs}\end{array}$ & $\begin{array}{c}28 \mathrm{a} \\
64,2 \mathrm{~h} \\
30 \mathrm{a}\end{array}$ & $\begin{array}{l}\text { I. E9 } \\
\text { ז. E8 }\end{array}$ & $\begin{array}{l}4,8 . E_{s} \\
4,8 . E_{s} \\
5,2 . \text { Es }\end{array}$ & $\begin{array}{l}\mathrm{I}, 60 . \mathrm{E}_{4} \\
\mathrm{I}, 60 . \mathrm{E}_{4} \\
\mathrm{I}, 74 . \mathrm{E}_{4}\end{array}$ & $\begin{array}{l}\text { 7,90. } \mathrm{E}_{4} \\
7,90 \cdot \mathrm{E}_{4} \\
8,60 \cdot \mathrm{E}_{4}\end{array}$ \\
\hline Total & & & $\mathrm{I}, 4^{8} . \mathrm{E}_{4}$ & $4,94 \cdot \mathrm{E}_{4}$ & $2,44 \cdot \mathrm{E}_{3}$ \\
\hline
\end{tabular}

Nota - I. E9 doit se lire $1.10^{-9}$. 